\title{
Ultra high temperature superfluidity in ultracold atomic Fermi gases with mixed dimensionality
}

\author{
Leifeng Zhang ${ }^{1}$, Jibiao $\mathrm{Wang}^{2}$, Yi Yu${ }^{1,3}$, and Qijin Chen ${ }^{1,4^{*}}$ \\ ${ }^{1}$ Department of Physics and Zhejiang Institute of Modern Physics, Zhejiang University, Hangzhou 310027, China; \\ ${ }^{2}$ Laboratory of Quantum Engineering and Quantum Metrology, School of Physics and Astronomy, Sun Yat-Sen University (Zhuhai Campus), \\ Zhuhai 519082, China; \\ ${ }^{3}$ College of Chemical Engineering, Zhejiang University of Technology, Hangzhou 310014, China; \\ ${ }^{4}$ Synergetic Innovation Center of Quantum Information and Quantum Physics, Hefei 230026, China
}

Received June 13, 2019; accepted July 19, 2019; published online September 2, 2019

Citation: L. F. Zhang, J. B. Wang, Y. Yu, and Q. J. Chen, Ultra high temperature superfluidity in ultracold atomic Fermi gases with mixed dimensionality, Sci. China-Phys. Mech. Astron. 63, 227421 (2020), https://doi.org/10.1007/s11433-019-9452-y

It has been an important goal to achieve higher or even room temperature superconductivity, since the discovery of high $T_{\mathrm{c}}$ superconductors in 1986, with a typical maximum transition temperature $T_{\mathrm{c}}$ of around $95 \mathrm{~K}$ at ambien pressure [1] or up to $164 \mathrm{~K}$ for the $\mathrm{Hg}$-based cuprates under high pressure [2]. The typical $T_{\mathrm{c}} / T_{\mathrm{F}}$ is only around 0.05 or less, where $T_{\mathrm{F}}$ denotes the Fermi temperature. There have been a few other families of superconductors, including the iron-based [3], heavy fermion [4] and organic superconductors [5]. Their maximum attainable $T_{\mathrm{c}} / T_{\mathrm{F}}$ has not been able to exceed that of the cuprates. Other notable superconductors include the recently discovered $\mathrm{H}_{2} \mathrm{~S}$ with a record high $T_{\mathrm{c}}=203 \mathrm{~K}$ under an enormous high pressure of $90 \mathrm{GPa}$ [6], and the monolayer $\mathrm{FeSe} / \mathrm{SrTiO}_{3}$ superconductors with a gap opening temperature up to $100 \mathrm{~K}$ [7]. The suggested conventional electron-phonon based pairing mechanism for both systems $[6,8]$ implies that their $T_{\mathrm{c}} / T_{\mathrm{F}}$ is very low. The very recently discovered superconductivity in magic angle twisted bilayer graphenes [9] has $T_{\mathrm{c}}=1.7 \mathrm{~K}$ with a near-flat band-width of $10 \mathrm{meV}$, leading to $T_{\mathrm{c}} / T_{\mathrm{F}} \sim 0.04$ (here we take $T_{\mathrm{F}}=5$ $\mathrm{meV} / \hbar)$, comparable to the cuprates.

There have been indications [10] for connections between high $T_{\mathrm{c}}$ superconductivity and BCS-Bose-Einstein condensa-

*Corresponding author (email: qchen@uchicago.edu) tion (BEC) crossover, the latter of which has a BEC asymptote of $T_{\mathrm{c}}=0.218 T_{\mathrm{F}}$ in three-dimensional (3D) continuum. It has been clear that with a $d$-wave pairing symmetry, high $T_{\mathrm{c}}$ cuprates fall in between the BCS and BEC regimes [11], with a pseudogap in the single-particle excitation spectrum.

Ultracold atomic Fermi gases have raised the hope for achieving a higher $T_{\mathrm{c}} / T_{\mathrm{F}}$. Indeed, BCS-BEC crossover in 3D Fermi gases has been realized experimentally since 2004 [12]. While a substantially higher BEC asymptote $T_{\mathrm{c}} / T_{\mathrm{F}}^{0}$ of 0.518 is possible in a trap (where $T_{\mathrm{F}}^{0}$ is the noninteracting global Fermi temperature), the maximum $T_{\mathrm{c}}$ in a homogeneous system occurs near unitarity, with a value around $T_{\mathrm{c}} / T_{\mathrm{F}} \sim 0.2$ in different theories and quantum Monte Carlo simulations as well as experimental measurements [13]. The local $T_{\mathrm{c}}(\mathbf{r}) / T_{\mathrm{F}}(\mathbf{r})$ in the trap never exceeds that of a homogeneous system. The increased BEC asymptote is due to an increased local density (and hence local $T_{\mathrm{F}}$ ) at the trap center.

The maximum $T_{\mathrm{c}} / T_{\mathrm{F}}$ for fermions on a lattice cannot surpass their continuum counterpart, since the lattice periodicity usually has a negative impact on the fermion and pair mobility and thus $T_{\mathrm{c}}$ [11].

In this letter, we propose that using a mixed dimensional setting, one may achieve ultra high $T_{\mathrm{c}}$ in units of $T_{\mathrm{F}}$. We find that, owing to the special features of mixed dimensions (MD), one can maintain a high $T_{\mathrm{c}}$, despite a tiny lattice hopping in- 
tegral $t$. We show that the maximum attainable $T_{\mathrm{c}} / T_{\mathrm{F}}$ may reach unity or even beyond the quantum degeneracy regime, well surpassing the maximum values in a pure 3D system or any known superfluids. This may shed light in the ultimate search for room temperature superconductivity.

Mixed dimensionality has been realized experimentally [14] in a Bose-Bose mixture of ${ }^{41} \mathrm{~K}-{ }^{87} \mathrm{Rb}$; only ${ }^{41} \mathrm{~K}$ atoms feel the lattice potential, leaving ${ }^{87} \mathrm{Rb}$ atoms moving freely in the 3D continuum. The species selective technique for the optical potential is also applicable for atomic Fermi gases.

MD was first investigated theoretically by Iskin and coworkers [15], for the BCS-BEC crossover of balanced fermion mixtures at zero $T$. A preliminary study of finite $T$ cases was reported [16]. Recently, a more systematic investigation of the pairing and superfluid phenomena at finite $T$ in MD has been reported for a balanced case [17]. The result seems to hint that $T_{\mathrm{c}}$ is higher for the cases of a larger lattice spacing $d$. However, one may also notice that these large $d$ situations are not readily accessible in simple experiments, with an overly large $t d^{2}$. It is important to investigate whether a greatly enhanced $T_{\mathrm{c}}$ is feasible with physically accessible parameters using MD.

Here we explore the effects of MD on the enhancement of Fermi level and show how this may lead to ultra high $T_{\mathrm{c}} / T_{\mathrm{F}}$ in atomic Fermi gases. Due to the high complexity caused by multiple tunable parameters, here we restrict ourselves to the population balanced case with equal masses, subject to a short-range attractive interaction of strength $g<0$. We shall consider the same dimensional setting as in the experiment of ref. [14], and use the same formalism based on a pairing fluctuation theory $[12,18]$ as in ref. [17] for MD. We refer to the lattice and 3D continuum components as spin up and spin down, respectively, and define the Fermi energy naturally as $E_{\mathrm{F}}=\hbar^{2} k_{\mathrm{F}}^{2} /(2 m)$, with $k_{\mathrm{F}}=\left(6 \pi^{2} n_{\downarrow}\right)^{1 / 3}$ being the Fermi momentum of the 3D component (we have set $\hbar=1$ ).

To keep the paper self-contained, we recapitulate the formalism. The band dispersions for the lattice and the 3D components are given by $\xi_{\mathbf{k} \uparrow}=\mathbf{k}_{\|}^{2} / 2 m+2 t\left[1-\cos \left(k_{z} d\right)\right]-\mu_{\uparrow}$ and $\xi_{\mathbf{k} \downarrow}=\mathbf{k}^{2} / 2 m-\mu_{\downarrow}$, respectively. Here $\mathbf{k}_{\|} \equiv\left(k_{x}, k_{y}\right)$, where $\mu_{\sigma}$ (with $\sigma=\uparrow, \downarrow$ ) are the fermionic chemical potentials. The one-band assumption is appropriate when the lattice band gap is experimentally tuned to be large compared with $E_{\mathrm{F}}$.

Both superfluid condensate, if present, and noncondensed pairs contribute to the fermion self-energy, and thus to the single particle excitation gap $\Delta$, via $\Delta^{2}=\Delta_{\mathrm{sc}}^{2}+\Delta_{\mathrm{pg}}^{2}$, where $\Delta_{\mathrm{sc}}$ and $\Delta_{\mathrm{pg}}$ are the superfluid order parameter and the pseudogap, respectively. Using the same four-vector notations as in refs. [12,17], the full Green's functions are given by

$$
\begin{aligned}
G_{\sigma}(K) & =\frac{u_{\mathbf{k}}^{2}}{\mathrm{i} \omega_{n}-E_{\mathbf{k} \sigma}}+\frac{v_{\mathbf{k}}^{2}}{\mathrm{i} \omega_{n}+E_{\mathbf{k} \bar{\sigma}}}, \quad\left|k_{z}\right|<\frac{\pi}{d}, \\
G_{\downarrow}(K) & =\frac{1}{\mathrm{i} \omega_{n}-\xi_{\mathbf{k} \downarrow}}, \quad\left|k_{z}\right|>\frac{\pi}{d},
\end{aligned}
$$

where $u_{\mathbf{k}}^{2}=\left(1+\xi_{\mathbf{k}} / E_{\mathbf{k}}\right) / 2, v_{\mathbf{k}}^{2}=\left(1-\xi_{\mathbf{k}} / E_{\mathbf{k}}\right) / 2, E_{\mathbf{k}}=$ $\sqrt{\xi_{\mathbf{k}}^{2}+\Delta^{2}}$, and $E_{\mathbf{k} \sigma}=E_{\mathbf{k}}+\zeta_{\mathbf{k} \sigma}, \xi_{\mathbf{k}}=\left(\xi_{\mathbf{k} \uparrow}+\xi_{\mathbf{k} \downarrow}\right) / 2, \zeta_{\mathbf{k} \sigma}=$ $\left(\xi_{\mathbf{k} \sigma}-\xi_{\mathbf{k} \bar{\sigma}}\right) / 2$.

The equations for the atomic number density $n=n_{\uparrow}+n_{\downarrow}$ and the number difference $\delta n=n_{\uparrow}-n_{\downarrow}=0$ are given by

$n=2 \sum_{\mathbf{k}}\left[v_{\mathbf{k}}^{2}+\bar{f}\left(E_{\mathbf{k}}\right) \frac{\xi_{\mathbf{k}}}{E_{\mathbf{k}}}\right]+\sum_{\left|k_{z}\right|>\pi / d} f\left(\xi_{\mathbf{k} \downarrow}\right)$,
$0=\sum_{\mathbf{k}}\left[f\left(E_{\mathbf{k} \uparrow}\right)-f\left(E_{\mathbf{k} \downarrow}\right)\right]-\sum_{\left|k_{z}\right|>\pi / d} f\left(\xi_{\mathbf{k} \downarrow}\right)$,

where $f(x)=1 /\left(\mathrm{e}^{x / T}+1\right)$, and $\bar{f}(x) \equiv \sum_{\sigma} f\left(x+\zeta_{\mathbf{k} \sigma}\right) / 2$.

In the superfluid state, the Thouless criterion leads to the gap [17]:

$\frac{m}{4 \pi a}=\frac{m_{\mathrm{eff}}}{4 \pi a_{\mathrm{eff}}}=\sum_{\mathbf{k}}\left[\frac{1}{2 \epsilon_{\mathbf{k}}}-\frac{1-2 \bar{f}\left(E_{\mathbf{k}}\right)}{2 E_{\mathbf{k}}}\right]$,

where $\epsilon_{\mathbf{k}}=\xi_{\mathbf{k}}+\mu$ with $\mu=\left(\mu_{\uparrow}+\mu_{\downarrow}\right) / 2$, and $a$ is the scattering length. Here the $3 \mathrm{D}$ equivalent effective mass, $m_{\mathrm{eff}}$, can be deduced from the trace of the inverse mass tensor, $\frac{1}{m_{\mathrm{eff}}}=\frac{5}{6 m}+\frac{1}{3} t d^{2}$. This then defines an effective scattering length $a_{\mathrm{eff}}$ such that $\frac{1}{k_{\mathrm{F}} a_{\mathrm{eff}}}=\frac{1}{k_{\mathrm{F}} a}\left(\frac{5}{6}+\frac{m}{3} t d^{2}\right)$. A plot of $a / a_{\mathrm{eff}}$ versus $k_{\mathrm{F}} d$ for $t / E_{\mathrm{F}}=0.1$ is shown in Figure $\mathrm{S} 1$ in Supporting information online.

The pair dispersion and related coefficients can be deduced via Taylor expanding the inverse $T$ matrix as: $t_{p g}^{-1}(Q) \approx$ $Z_{1}\left(\mathrm{i} \Omega_{l}\right)^{2}+Z\left(\mathrm{i} \Omega_{l}-\Omega_{\mathbf{q}}\right)$, where $\Omega_{\mathbf{q}}=q_{\|}^{2} / 2 M_{\|}^{*}+q_{z}^{2} / 2 M_{z}^{*}$ in the superfluid phase [12], with pair masses $M_{\|}^{*}$ and $M_{z}^{*}$ in the $x y$ and $z$ directions, respectively.

The pseudogap $\Delta_{p g}$ is related to the density of pairs, via

$\Delta_{p g}^{2}=\sum_{\mathbf{q}_{\|}} \sum_{\left|q_{z}\right|<\pi / d} \frac{b\left(\tilde{\Omega}_{\mathbf{q}}\right)}{Z \sqrt{1+4 \frac{Z_{1}}{Z} \Omega_{\mathbf{q}}}}$,

where $b(x)$ is the Bose distribution function and $\tilde{\Omega}_{\mathbf{q}}=$ $Z\left\{\sqrt{1+4 Z_{1} \Omega_{\mathbf{q}} / Z}-1\right\} / 2 Z_{1}$ is the pair dispersion.

The closed set of eqs. (2)-(5) will be solved for $T_{\mathrm{c}}$ (and $\Delta_{\mathrm{pg}}$ and $\mu_{\sigma}$ at $T_{\mathrm{c}}$ ), by setting the order parameter $\Delta_{\mathrm{sc}}=0$.

The solution for $T_{\mathrm{c}}$ in the deep BEC regime can be simplified dramatically, where the $Z_{1} \Omega^{2}$ term is negligible. It can be shown that $M_{\|}^{*}=M_{z}^{*}=2 m$ so that $\Omega_{\mathbf{q}}=\frac{q^{2}}{4 m}$. And $T_{\mathrm{c}}$ is determined by the pseudogap eq. (5), which reduces to

$\frac{n}{2}=\sum_{\mathbf{q}_{\|} \mid} \sum_{\left|q_{z}\right| \leq \pi / d} b\left(\Omega_{\mathbf{q}}\right)$.

The BEC asymptote for $T_{\mathrm{c}}$ increases as $d$ becomes larger; a larger $d$ leads to a more reduced phase space in the $\hat{z}$ direction, and thus needs a higher $T_{\mathrm{c}}$ to excite pairs into higher $q_{\|}$states, in order to satisfy eq. (6).

As shown in Figure 1, large $d$ can substantially push up the Fermi level, $\mu_{\uparrow}$, of the lattice component in the noninteracting 
limit, leading to a "disk"-like filling in momentum space, as exemplified by Figure $\mathrm{S} 2$ for $t=0.01 E_{\mathrm{F}}$ and $k_{\mathrm{F}} d=8$.

Shown in Figure 2 is $T_{\mathrm{c}}$ versus $1 / k_{\mathrm{F}} a_{\mathrm{eff}}$ at a realistic value of $2 m t d^{2}=0.16$, but for a series of values of $k_{\mathrm{F}} d$ from 1 up to 55. Each curve has a maximum $T_{\mathrm{c}}, T_{\mathrm{c}}^{\max }$, and a BEC asymptote $T_{\mathrm{c}}^{\mathrm{BEC}}$ in the large $1 / k_{\mathrm{F}} a_{\mathrm{eff}}$ limit. As plotted in the inset of Figure 3, both $T_{\mathrm{c}}^{\max }$ and $T_{\mathrm{c}}^{\mathrm{BEC}}$ increase with $k_{\mathrm{F}} d$ almost linearly, without an upper bound. At $k_{\mathrm{F}} d=55$, $T_{\mathrm{c}}^{\max } / T_{\mathrm{F}}=0.995 \approx 1$, namely, the maximum $T_{\mathrm{c}}$ is close to $T_{\mathrm{F}}$, and the BEC asymptote $T_{\mathrm{c}}^{\mathrm{BEC}}$ has risen up to $0.72 T_{\mathrm{F}}$. Other quantities including $\Delta, 1 / k_{\mathrm{F}} a_{\text {eff }}$ and $\mu_{\sigma}$ at the maximum $T_{\mathrm{c}}$ points are plotted in Figure S3.

Note that $T_{\mathrm{c}}$ divided by the noninteracting $\mu_{\uparrow}$ from Figure 1 will not exhibit such an increase with $d$. However, unlike a pure lattice case, the MD sitting provides the noninteracting $E_{\mathrm{F}}$ of the $3 \mathrm{D}$ component as a natural energy scale, so that the noninteracting $\mu_{\uparrow}$ becomes a variable that can be tuned via $t$ and $d$. Alternatively, one may think of the increase

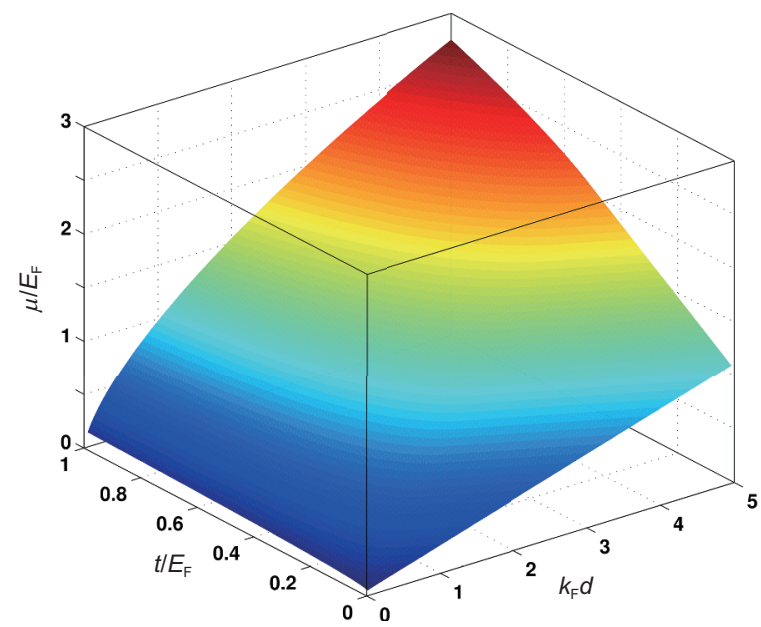

Figure 1 (Color online) Evolution of the chemical potential $\mu_{\uparrow}$ of the lattice component, as a function of $t$ and $d$. $\mu_{\uparrow}$ stays low for small $d$ and becomes elevated for large $d$.

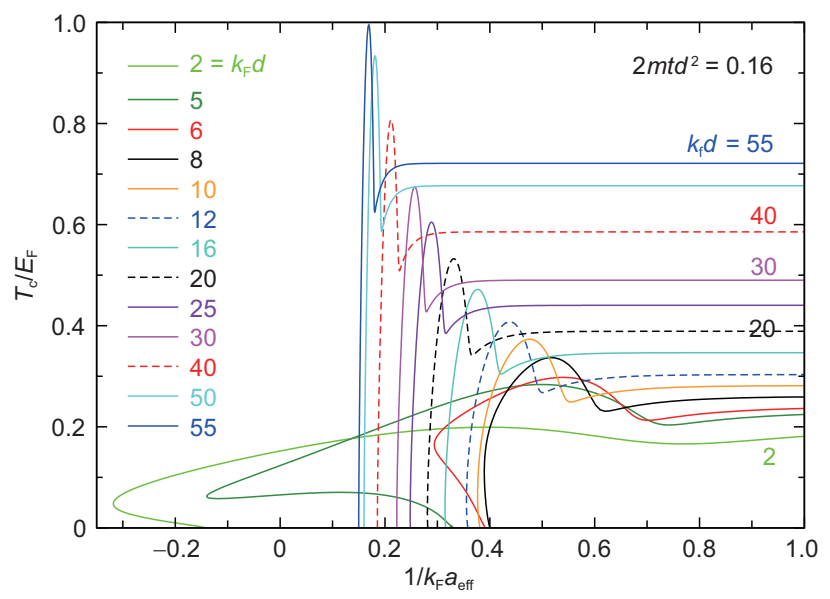

Figure 2 (Color online) Behavior of $T_{\mathrm{c}}$ as functions of $1 / k_{\mathrm{F}} a_{\mathrm{eff}}$ at fixed $2 m t d^{2}=0.16$, but for different values of $k_{\mathrm{F}} d$ from 1 up to 55 . of $T_{\mathrm{c}}$ as compared with a given Fermi gas of the same atom density in $3 \mathrm{D}$ continuum.

To understand the evolution of $\mu_{\uparrow}$ at large $d$, we show in Figure 3 the momentum distribution $n_{\downarrow}\left(\mathbf{k}_{\|}=0, k_{z}\right)$ of the 3D components for $k_{\mathrm{F}} d=4$ and $t / E_{\mathrm{F}}=0.01$ along the $k_{z}$ axis with different pairing strengths in the unitary and near BEC regimes. As $1 / k_{\mathrm{F}} a$ increases from unitarity, the spectral weight outside the first BZ decreases rapidly, and essentially vanishes for $1 / k_{\mathrm{F}} a=0.8$. However, due to lack of sharp features, the corresponding in-plane momentum distribution $n_{\downarrow}\left(\mathbf{k}_{\|}, k_{z}=0\right)$ of the $3 \mathrm{D}$ component in the $k_{z}=0$ plane, as shown in Figure S4, does not look qualitatively much different from its pure 3D counterpart.

An inspection of $n_{\downarrow}\left(\mathbf{k}_{\|}=0, k_{z}\right)$ at the maximum $T_{\mathrm{c}}$ points for a series of $d$ values, as shown in Figure S5, reveals that the spectral weight outside the first BZ is not necessarily zero in order to reach the maximum $T_{\mathrm{c}}$; finite $T$ Fermi surface smearing allows a considerable mismatch in momentum distributions between the two pairing components. The shift of the spectral weight in the in-plane momentum distribution, as shown in Figure S6(a), toward higher $k_{\|}$with increasing $d$ can be made apparent through the higher order moments, $k_{\|}^{n} n_{\downarrow}\left(\mathbf{k}_{\|}, k_{z}=0\right)$. As shown in Figure S6(b) for $n=2$, both the peak location and the peak height increase with $d$.

We also investigate the effect of a varying hopping matrix element $t$ on $T_{\mathrm{c}}$ with a fixed $d$. For large $d$, the experimentally accessible range of $t$ is fairly small, with the restriction $2 m t d^{2}<1$. Plotted in Figure 4 is $T_{\mathrm{c}}$ versus $1 / k_{\mathrm{F}} a$ for $k_{\mathrm{F}} d=20$ with different $t / E_{\mathrm{F}}$ from 0.0004 up to 0.1 . Note that except for the lowest two $t$ values, the rest curves are not readily accessible in experiment. Nonetheless, except for a roughly parallel shift of one another, decreasing $t / E_{\mathrm{F}}$ from 0.1 down to 0.0004 or smaller barely affect the $T_{\mathrm{c}}$ curves. A

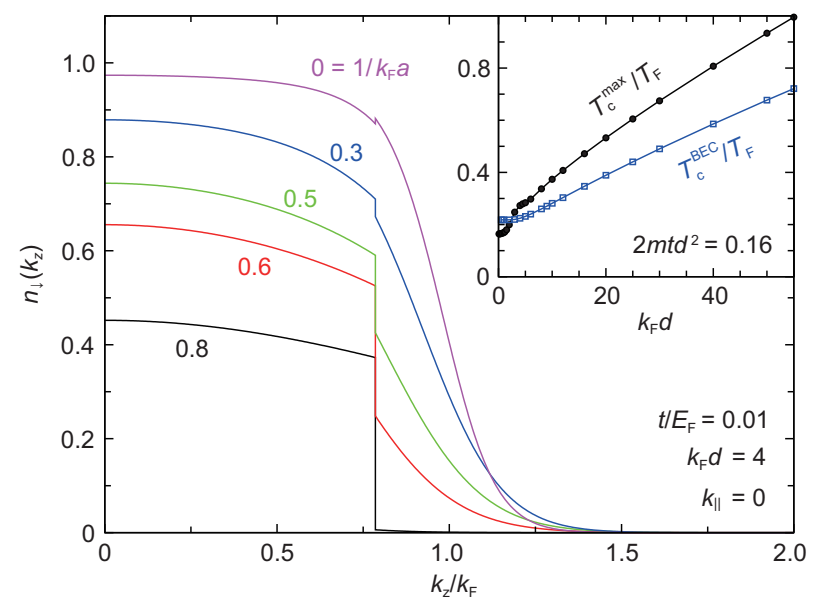

Figure 3 (Color online) Momentum distribution $n\left(\mathbf{k}_{\|}=0, k_{z}\right)$ of the 3D component along the $k_{z}$ axis for different pairing strength, characterized by $1 / k_{\mathrm{F}} a$, with $t / E_{\mathrm{F}}=0.01$ and $k_{\mathrm{F}} d=4$. Here we fix the in-plane momentum $\mathbf{k}_{\|}=0$. Upon entering the BEC regime, the occupation for $k_{z}>\pi / d$ decreases rapidly. Shown in the inset are $T_{\mathrm{c}}^{\max } / T_{\mathrm{F}}$ and $T_{\mathrm{c}}^{\mathrm{BEC}} / T_{\mathrm{F}}$ versus $k_{\mathrm{F}} d$ from Figure 2 . 


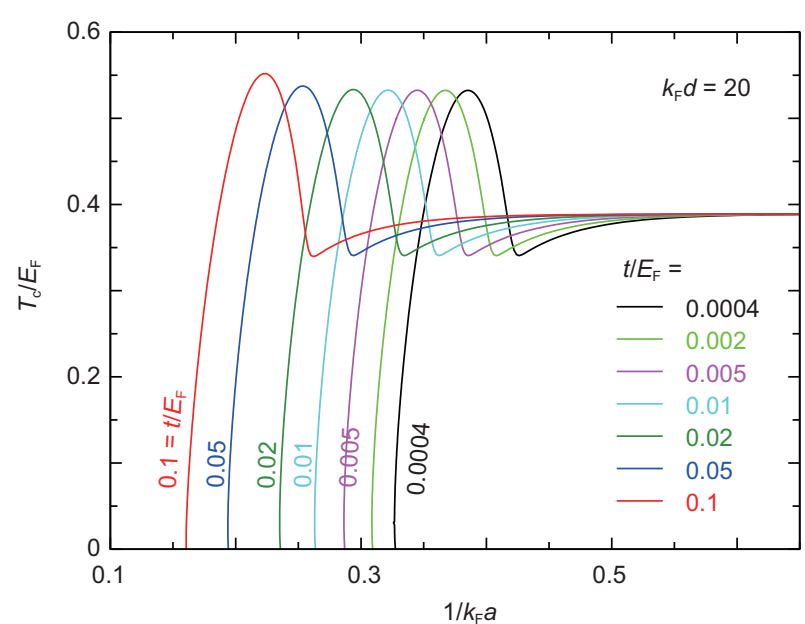

Figure 4 (Color online) Effects of $t$ on the behavior of $T_{\mathrm{c}}$ for fixed $k_{\mathrm{F}} d=20$ with different $t / E_{\mathrm{F}}$ from 0.0004 up to 0.1 .

replot of Figure 4 as a function of $1 / k_{\mathrm{F}} a_{\mathrm{eff}}$ is given in Figure $\mathrm{S} 7$, where the $T_{\mathrm{c}}$ curves are horizontally rescaled by different factors.

We note that it may not be easy to control very large $d$ values experimentally. In addition, the pairing gap at the maximum $T_{\mathrm{c}}$ for the $d=50$ case is huge, as shown in Figure S3(a). This likely points to the need to include higher energy bands in the lattice dimension. Nevertheless, we argue that as long as the band gap is large, the contributions from the higher energy bands will only cause a secondary, quantitative correction to $T_{\mathrm{c}}$. It shall remain valid that a large $d$ in MD will substantially enhance $T_{\mathrm{c}}$.

Experimentally a Fermi-Fermi mixture may be needed in order to achieve MD. Nonetheless, the Fermi momentum does not depend on the atomic mass $m$ or hopping integral $t$. Therefore, upon pairing, one can still achieve a perfect Fermi surface match, as long as the populations are balanced. The mechanism for the enhancement of $T_{\mathrm{c}}$ remains valid. A close match between masses may occur for pairing between two isotopic atoms, such as ${ }^{161}$ Dy and ${ }^{163} \mathrm{Dy}$. Detailed quantitative influences of mass imbalance (and other factors such as dipolar interactions) will be investigated in future studies.

It should be emphasized that our findings about the enhancement of $T_{\mathrm{c}}$ via MD are essentially independent of the details of our pairing fluctuation theory. Alternative theories such as the Nozeres-Schmitt-Rink [19] and FLEX approximations [20] of the $T$-matrix theories should yield qualitatively similar results.

Finally we note that a key difference between the MD and pure lattice cases is that the effective pair mass $M_{z}$ in the lattice direction is drastically different in the BEC asymptote. For the former case, $M_{z}$ approaches $2 m$, since the total kinetic energy of the two pairing atoms is dominated by the $3 \mathrm{D}$ component so that pairs never become local around the lattice sites. This is an unusual feature of mixed dimensionality. In contrast, in the pure lattice case, pairs move mainly via vir- tual ionization [19]. This leads to an effective pair hopping integral $t_{\mathrm{B}} \sim-t^{2} / g$ so that $M_{z} \sim 1 / t_{\mathrm{B}} \sim|g|$ becomes heavy in the BEC regime. We emphasize that this difference is the key to understand why for the tiny $t / E_{\mathrm{F}}=5.3 \times 10^{-5}$ in the case of $k_{\mathrm{F}} d=55, T_{\mathrm{c}}$ is hardly suppressed. This also implies that the BEC asymptote $T_{\mathrm{c}}^{\mathrm{BEC}}$ is governed by $d$, whereas $t$ becomes essentially irrelevant.

In summary, one may achieve ultra high temperature superfluids using such an MD setting with a large $d$, with a greatly enhanced $T_{\mathrm{c}}$, all the way up to (or even beyond) the quantum degeneracy temperature $T_{\mathrm{F}}$. And the BEC asymptote $T_{\mathrm{c}}^{\mathrm{BEC}}$ is pushed up dramatically as well.

This work was supported by the National Natural Science Foundation of China (Grant Nos. 11274267, and 11774309), the National Basic Research Program of China (Grant No. 2012CB927404), Natural Science Foundation of Zhejiang Province of China (Grant No. LZ13A040001). We thank B. Anderson, Y. A. Chen, K. Y. Gao, A. J. Leggett, K. Levin, G. Watanabe, B. Yan and X. C. Yao for useful discussions. Part of this work was completed while $Q C$ was visiting the University of Chicago.

\section{Supporting Information}

The supporting information is available online at phys.scichina.com and http://link.springer.com/journal/11433. The supporting materials are published as submitted, without typesetting or editing. The responsibility for scientific accuracy and content remains entirely with the authors.

1 T. Timusk, and B. Statt, Rep. Prog. Phys. 62, 61 (1999).

2 L. Gao, Y. Y. Xue, F. Chen, Q. Xiong, R. L. Meng, D. Ramirez, C. W. Chu, J. H. Eggert, and H. K. Mao, Phys. Rev. B 50, 4260 (1994).

3 Y. Kamihara, T. Watanabe, M. Hirano, and H. Hosono, J. Am. Chem. Soc. 130, 3296 (2008)

4 F. Steglich, J. Aarts, C. D. Bredl, W. Lieke, D. Meschede, W. Franz, and H. Schäfer, Phys. Rev. Lett. 43, 1892 (1979).

5 R. H. McKenzie, Science 278, 820 (1997).

6 A. P. Drozdov, M. I. Eremets, I. A. Troyan, V. Ksenofontov, and S. I. Shylin, Nature 525, 73 (2015), arXiv: 1506.08190.

7 J. F. Ge, Z. L. Liu, C. Liu, C. L. Gao, D. Qian, Q. K. Xue, Y. Liu, and J. F. Jia, Nat. Mater. 14, 285 (2015), arXiv: 1406.3435.

8 D. H. Lee, Chin. Phys. B 24, 117405 (2015).

9 Y. Cao, V. Fatemi, S. Fang, K. Watanabe, T. Taniguchi, E. Kaxiras, and P. Jarillo-Herrero, Nature 556, 43 (2018), arXiv: 1803.02342.

10 Y. J. Uemura, Phys. C-Supercond. 282-287, 194 (1997).

11 Q. J. Chen, I. Kosztin, B. Jankó, and K. Levin, Phys. Rev. B 59, 7083 (1999).

12 Q. J. Chen, J. Stajic, S. N. Tan, and K. Levin, Phys. Rep. 412, 1 (2005).

13 Q. J. Chen, Phys. Rev. A 86, 023610 (2012), arXiv: 1109.5327.

14 G. Lamporesi, J. Catani, G. Barontini, Y. Nishida, M. Inguscio, and F. Minardi, Phys. Rev. Lett. 104, 153202 (2010), arXiv: 1002.0114.

15 M. Iskin, and A. L. Subaşl, Phys. Rev. A 82, 063628 (2010), arXiv: 1004.3111.

16 X. S. Yang, B. B. Huang, and S. L. Wan, Eur. Phys. J. B 83, 445 (2011), arXiv: 1104.2375.

17 L. F. Zhang, Y. M. Che, J. B. Wang, and Q. J. Chen, Sci. Rep. 7, 12948 (2017), arXiv: 1710.00200.

18 Q. J. Chen, I. Kosztin, B. Jankó, and K. Levin, Phys. Rev. Lett. 81, 4708 (1998).

19 P. Nozières, and S. Schmitt-Rink, J. Low Temp. Phys. 59, 195 (1985).

20 N. E. Bickers, D. J. Scalapino, and S. R. White, Phys. Rev. Lett. 62, 961 (1989). 